\title{
Photon Generalized Parton Distributions
}

the date of receipt and acceptance should be inserted later

\begin{abstract}
We present a calculation of the generalized parton distributions of the photon using overlaps of photon light-front wave functions.
\end{abstract}

Keywords Photon Structure · Generalized Parton distributions

\section{Introduction}

Generalized parton distributions (GPDs) of the proton are objects of interest both theoretically and in experiments since some time. Among many other aspects that make them interesting, these are a possible way to get information about the orbital angular momentum of the quarks inside the proton (GPDs) [1]. These appear in the amplitudes of certain exclusive processes for example deeply virtual Compton scattering (DVCS), $e p \rightarrow e p^{\prime} \gamma$ where there is a finite momentum transfer between the intial and the final proton and a real photon is observed in the final state. It has been shown that in the Bjorken regime, the amplitude factorizes and can be written in terms of a hard perturbative part and the soft part parametrized in terms of the GPDs. Unlike the ordinary parton distributions (pdfs), which can be expressed as forward matrix elements of certain operators, GPDs are off-forward matrix elements of such operators, thus they are richer in content than the pdfs. GPDs are being extracted from data on DVCS and hard exclusive meson production processes at DESY, COMPASS and JLab experiments. Apart from $x$, which is the momentum fraction of the active quark in the proton, GPDs depend on the skewness $\zeta$, which is related to the longitudinal momentum transfer and the momentum transfer squared $t$ at a given scale. The second moment of the sum of the GPDs $H(x, \zeta, t)$ and $E(x, \zeta, t)$ is related to the part of the nucleon spin carried by the quarks and antiquarks. In the forward limit the GPD $H(x, 0,0)$ gives the pdf $q(x)$. In [2] it was shown that after a Fourier transform of the GPDs with respect to the momentum transfer in the transverse direction $\Delta_{\perp}$, one gets the distribution of partons in the transverse position space or impact parameter plane. When the longitudinal momentum transfer is zero, this gives the distribution of partons in the nucleon in the transverse plane. They are called impact parameter dependent parton distributions (ipdpdfs) $q\left(x, b^{\perp}\right)$. In fact they obey certain positivity constraints and can be interpreted as probability densities. In some other works, GPDs have been investigated in three dimensional position space. In [3] DVCS amplitude was expressed in boost invariant longitudinal position space conjugate to the skewness $\zeta$ and a pattern similar to the diffraction pattern in optics was observed.

Deeply virtual Compton scattering (DVCS) $\gamma^{*} \gamma \rightarrow \gamma \gamma$ on a photon target was considered in [4] in the kinematic region of large virtuality $\left(Q^{2}\right)$ but small squared momentum transfer $(-t)$ between the

Asmita Mukherjee

Department of Physics

Indian Institute of Technology Bombay

Powai, Mumbai 400076, India 

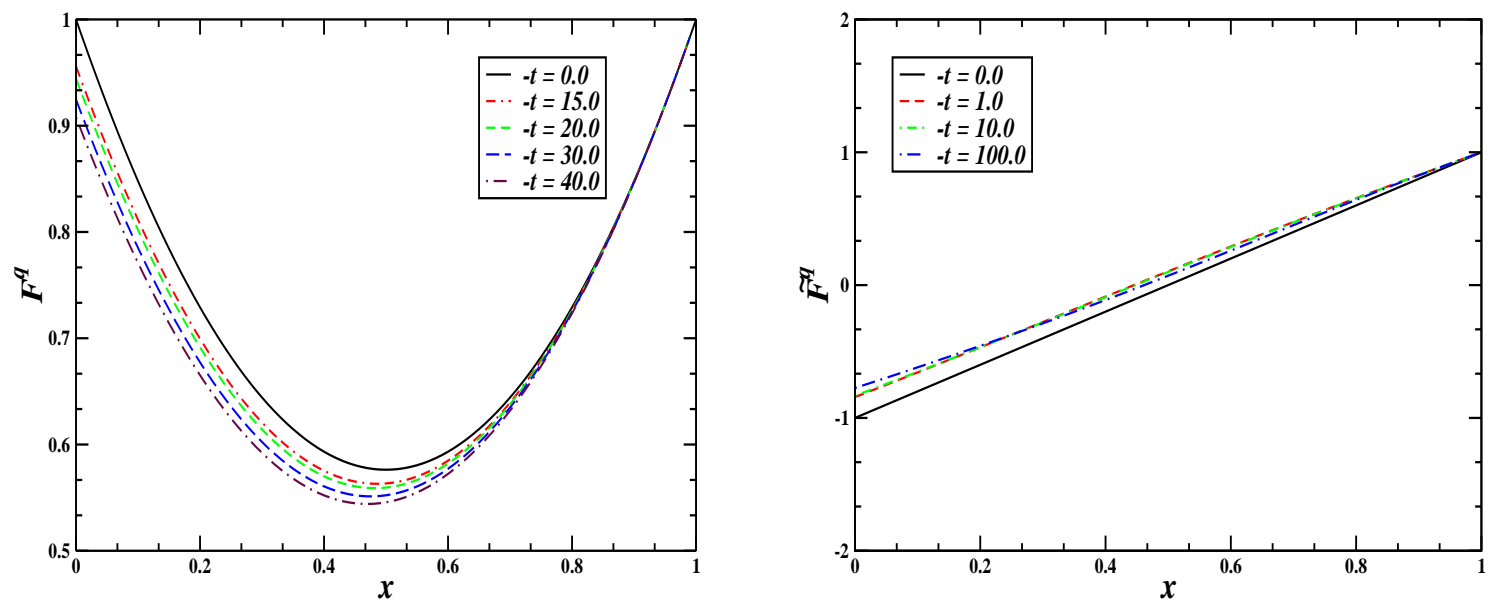

Fig. 1 (Color online) Plots of unpolarized GPD $F^{q}$ and polarized GPD $\tilde{F}^{q}$ vs $x$ for fixed values of $-t$ in $M e V^{2}$ $\Lambda=2 \mathrm{GeV}$. In both plots the normalization factor is chosen to compare with [4] when $t=0$.

initial and final (real) photon. The result was interpreted at leading logarithmic order as a factorized form of the scattering amplitude in terms of a hard handbag diagram and the generalized parton distributions of the photon. This was shown at leading order in $\alpha$ and zeroth order in $\alpha_{s}$ when the momentum transfer was purely in the longitudinal direction. The GPDs show logarithmic scale dependence already in parton model, like the photon structure functions. They are interesting as they can be calculated in perturbation theory and can act as theoretical tools to understand the basic properties of GPDs like polynomiality and positivity. Beyond leading logarithmic order one would need to include the non-pointlike hadronic contribution which will be model dependent. It is interesting to access the partonic structure of the photon probed in high energy processes, and photon GPDs can shed more light on the partonic content of the photon.

\section{Generalized Parton Distributions of the Photon}

The GPDs for the photon are defined as [4]:

$$
\begin{gathered}
F^{q}=\int \frac{d y^{-}}{8 \pi} e^{\frac{-i P^{+} y^{-}}{2}}\left\langle\gamma\left(P^{\prime}\right)\left|\bar{\psi}(0) \gamma^{+} \psi\left(y^{-}\right)\right| \gamma(P)\right\rangle ; \\
\tilde{F}^{q}=\int \frac{d y^{-}}{8 \pi} e^{\frac{-i P^{+} y^{-}}{2}}\left\langle\gamma\left(P^{\prime}\right)\left|\bar{\psi}(0) \gamma^{+} \gamma^{5} \psi\left(y^{-}\right)\right| \gamma(P)\right\rangle .
\end{gathered}
$$

$F^{q}$ contributes when the photon is unpolarized and $\tilde{F}^{q}$ is the contribution from the polarized photon. The second is extracted from the terms containing $\epsilon_{\lambda}^{2} \epsilon_{\lambda}^{1 *}-\epsilon_{\lambda}^{1} \epsilon_{\lambda}^{2 *}$ in the amplitude [4]. $\lambda$ is the helicity of the photon. We consider the terms where the photon helicity is not flipped. We have chosen the light-front gauge $A^{+}=0 . F^{q}$ and $\tilde{F}^{q}$ can be calculated using the Fock space expansion of the state. Two-particle light-front wave functions of the photon can be calculated analytically in perturbation theory and are boost invariant.

We calculate the above matrix elements using the overlaps of photon light-front wave functions at leading order in $\alpha$ and zeroth order in $\alpha_{s}$, keeping leading logarithmic terms. However, we keep the quark mass terms in the vertex. The results are scale dependent, this scale dependence in our approach comes from the upper limit of the transverse momentum integration $\Lambda=Q$. There is a lower cutoff on the transverse momentum, which can be taken to zero as long as the quark mass is nonzero. Leading order evolution of the photon GPDs has been calculated in [4] for non-zero $\zeta$. The mass terms in the vertex give subdominant contributions. The analytic expressions for the photon GPDs can be found in [5]. Here we give the numerical results.

In analogy with the impact parameter dependent parton distribution of the proton, we introduce the same for the photon. By taking a Fourier transform with respect to the transverse momentum 

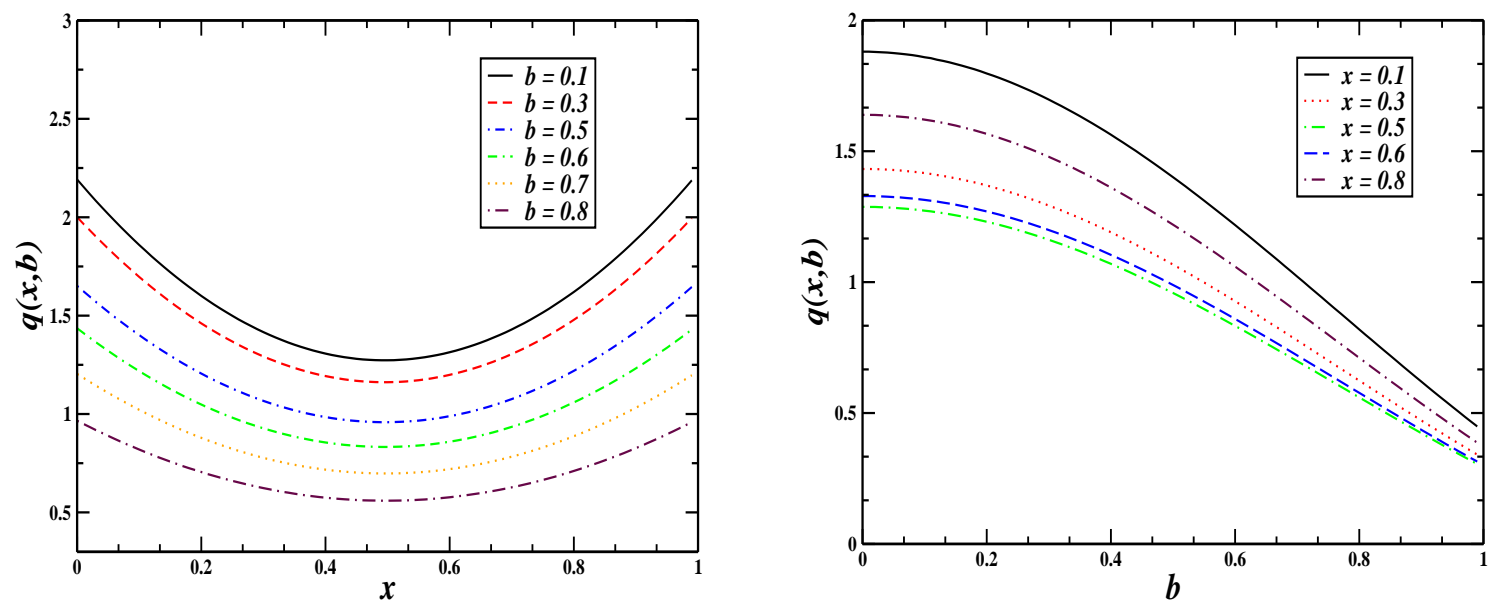

Fig. 2 (Color online) Plot of impact parameter dependent pdf $q(x, b)$ vs $x$ for fixed $b$ values and $q(x, b)$ vs $b$ for fixed values of $x$ where we have taken $\Lambda=2 \mathrm{GeV}$ and $\Delta_{\max }=3 \mathrm{MeV}$ where $\Delta_{\max }$ is the upper limit in the $\Delta$ integration. $b$ is in $\mathrm{MeV}^{-1}$ and $q(x, b)$ is in $\mathrm{MeV}^{2}$.

transfer $\Delta^{\perp}$ we get the GPDs in the transverse impact parameter space.

$$
\begin{aligned}
q\left(x, b^{\perp}\right) & =\frac{1}{(2 \pi)^{2}} \int d^{2} \Delta^{\perp} e^{-i \Delta^{\perp} \cdot b^{\perp}} F^{q} \\
& =\frac{1}{2 \pi} \int \Delta d \Delta J_{0}(\Delta b) F^{q} ; \\
\tilde{q}\left(x, b^{\perp}\right) & =\frac{1}{(2 \pi)^{2}} \int d^{2} \Delta^{\perp} e^{-i \Delta^{\perp} \cdot b^{\perp}} \tilde{F}^{q} \\
& =\frac{1}{2 \pi} \int \Delta d \Delta J_{0}(\Delta b) \tilde{F}^{q} ;
\end{aligned}
$$

where $J_{0}(z)$ is the Bessel function; $\Delta=\left|\Delta^{\perp}\right|$ and $b=\left|b^{\perp}\right|$. In the numerical calculation, we have introduced a maximum limit $\Delta_{\max }$ of the $\Delta$ integration which we restrict to satisfy the kinematics $-t<<Q^{2}[3 ;$; $]$ ]. $q\left(x, b^{\perp}\right)$ gives the distribution of partonsin the photon. Like the proton, this interpretation holds in the infinite momentum frame and there is no relativistic correction to this identification in light-front formalism.

We have plotted the unpolarized GPD $F^{q}$ and the polarized GPD $\tilde{F}^{q}$ for the photon respectively in Fig. 1 as functions of $x$ and for different values of $t=-\left(\Delta^{\perp}\right)^{2}$. In all plots we took the momentum transfer to be purely in the transverse direction. We took the mass of the quark as well as antiquark to be $m=3.3 \mathrm{MeV} ; \Lambda=Q=2 \mathrm{GeV}$. We divided the GPDs by the normalization constant to compare with [4] in the limit of zero $t$. At larger values of $x$, most of the momentum is carried by the quark in the photon and the GPDs become independent of $t$. The Fourier transform (FT) of the unpolarized GPD $F^{q}$ is plotted in Fig. 2 and polarized GPD $\tilde{F}^{q}$ is plotted in Fig. 3. In all plots we have taken $0<x<1$ for which the contribution comes from the active quark in the photon $(q \bar{q})$. The smearing in $b^{\perp}$ space reveals the partonic substructure of the photon and shows its 'shape' in transverse position space. The behavior of the photon GPDs in impact parameter space is qualitatively different from phenomenological models of proton GPDs [6]. In the phenomenological parametrization of proton GPDs where spectator model with Regge-type modification was used, the $u$ quark GPDs increase with increasing $x$ for fixed $b$, reaches a maximum, then decrease. The peak decreases with increasing $b$ [6]. In the case of a photon the distribution in $b$ space purely reveals the internal $q \bar{q}$ structure of the photon. Near $x \approx 1 / 2$ the peak in $b$ space is very broad which means that the parton distribution is more dispersed when the $q$ and $\bar{q}$ share almost equal momenta. The slope of the polarized distribution decreases for higher $b$. The sign of the GPD changes at $x=1 / 2$, at which point the GPD and the pdf in impact parameter space becomes zero. 

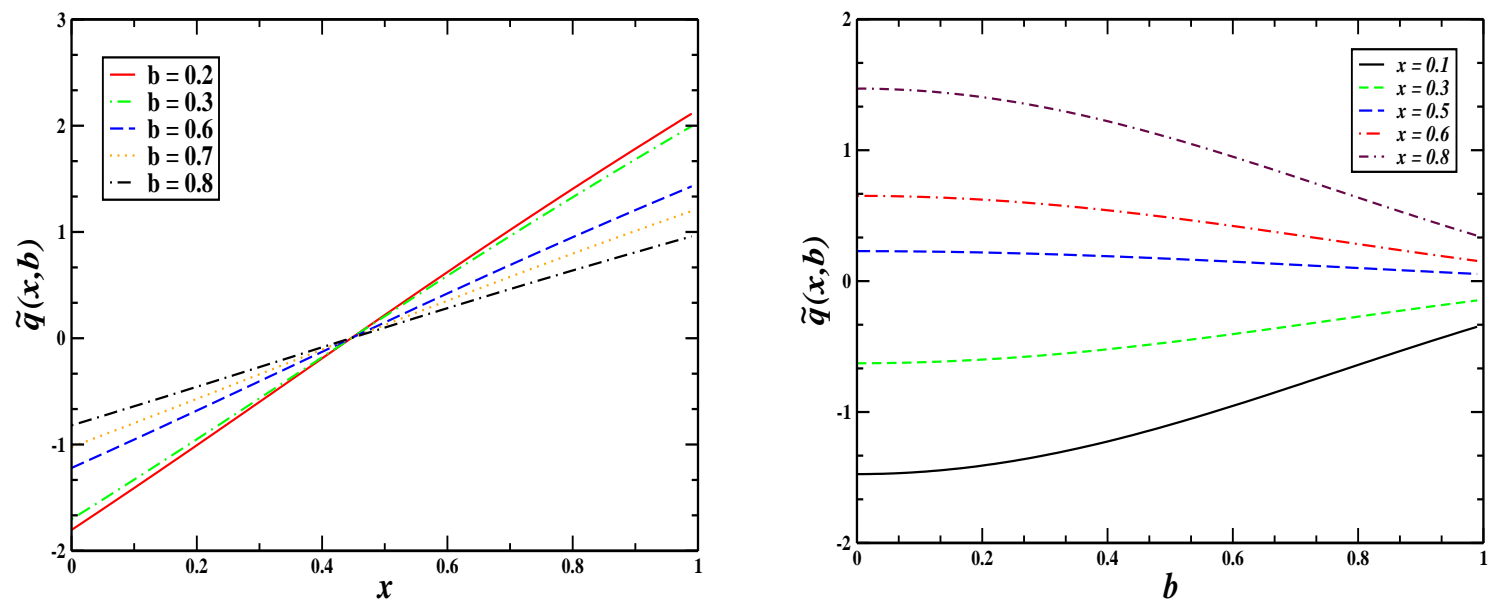

Fig. 3 (Color online) Plot of impact parameter dependent pdfs $\tilde{q}(x, b)$ vs $x$ for fixed $b$ values and $\tilde{q}(x, b)$ vs $b$ for fixed values of $x$ where we have taken $\Lambda=2 \mathrm{GeV}$ and $\Delta_{\max }=3 \mathrm{MeV}$ where $\Delta_{\max }$ is the upper limit in the $\Delta$ integration. $b$ is in $\mathrm{MeV}^{-1}$ and $\tilde{q}(x, b)$ is in $\mathrm{MeV}^{2}$.

\section{Conclusion}

We discussed a calculation of the generalized parton distributions of the photon, both polarized and unpolarized. We took the momentum transfer in the transverse direction is non-zero. We calculated at zeroth order in $\alpha_{s}$ and leading order in $\alpha$; also at leading logarithmic order; we kept the mass terms at the vertex. We took the skewness to be zero. Taking a Fourier transform with respect to the momentum transfer in the transverse direction we obtain impact parameter dependent parton distribution of the photon. These give a unique picture of the photon in transverse position space.

\section{Acknowledgments}

This work is supported by BRNS grant Sanction No. 2007/37/60/BRNS/2913 dated 31.3.08, Govt. of India. AM Thanks the organizers of Light Cone 2011 for the kind invitation and support.

\section{References}

1. For reviews on generalized parton distributions, and DVCS, see M. Diehl, Phys. Rept, 388, 41 (2003); A. V. Belitsky and A. V. Radyushkin, Phys. Rept. 418 1, (2005); K. Goeke, M. V. Polyakov, M. Vanderhaeghen, Prog. Part. Nucl. Phys. 47, 401 (2001); S. Boffi, B. Pasquini, Riv.Nuovo Cim.30:387,2007.

2. M. Burkardt, Int. J. Mod. Phys. A 18, 173 (2003); M. Burkardt, Phys. Rev. D 62, 071503 (2000), Erratumibid, D 66, 119903 (2002); J. P. Ralston and B. Pire, Phys. Rev. D 66, 111501 (2002).

3. S. J. Brodsky, D. Chakrabarti, A. Harindranath, A. Mukherjee and J. P. Vary, Phys. Lett. B 641, 440 (2006); Phys. Rev. D 75, 014003 (2007).

4. S. Friot, B. Pire, L. Szymanowski, Phys. Lett. 645153 (2007).

5. A. Mukherjee and S. Nair, arXiv:1105.5299 [hep-ph].

6. D. Chakrabarti, R. Manohar, A. Mukherjee, Phys. Lett. B 682, 428 (2010); R. Manohar, A. Mukherjee, D. Chakrabarti, Phys.Rev.D83, 014004,(2011). 